\title{
Ball and Cylinder Interferometer
}

\author{
J. B. Saunders, Sr. \\ Institute for Basic Standards, National Bureau of Standards, Washington, D.C. 20234
}

(March 24, 1972)

\begin{abstract}
An interferometer is described that permits the comparison of diameters of balls and cylinders with an etalon. The specimen is located in the cavity of the etalon. Interference is produced by light reflected from each surface of the etalon and the adjacent surface of the specimen. The two pairs of interfering beams of light (one pair from each side of the etalon) are received by the same eyepiece or camera. A shutter permits alternate viewing of the two interferograms. Magnification and contrast of the fringes are obtained by a suitable choice of power and separations of components of the system. After the prime dimension of the etalon is determined, it can be subsequently rechecked with the single mode (stabilized) laser, used with this interferometer, without removing the etalon from the assembly. A method is provided for analyzing the results of this interferometer.
\end{abstract}

Key words: Density standards; interferometer; volume of spheres.

\section{Introduction}

This interferometer was designed primarily for measuring the volumes of near perfect spheres that are intended to represent standards for density. The spherical form was chosen because it is believed to permit the highest accuracy for volumetric determination.

Measurement of the geometry of balls and cylinders has usually been made with electrical and mechanical probes. The interferometer method, described below, is believed to provide greater accuracy than can be obtained by other methods. It also eliminates the necessity of physical contact between the surface and other elements, except for gravitational support.

This interferometer permits the measurement of the minimum separation $\left(d_{1}\right.$ and $d_{2}$, fig. 1$)$ of the specimen from each of the etalon faces, $E_{1}$ and $E_{2}$. If the cross section of the specimen approximates a circle, the average of several diameters (whose directions are uniformly spaced) approximates the diameter of a circle whose area equals that of the section. Likewise, if the shape of the specimen approximates a sphere, the average of many equally spaced (angularwise) diameters approximates the diameter of a sphere whose volume equals that of the specimen.

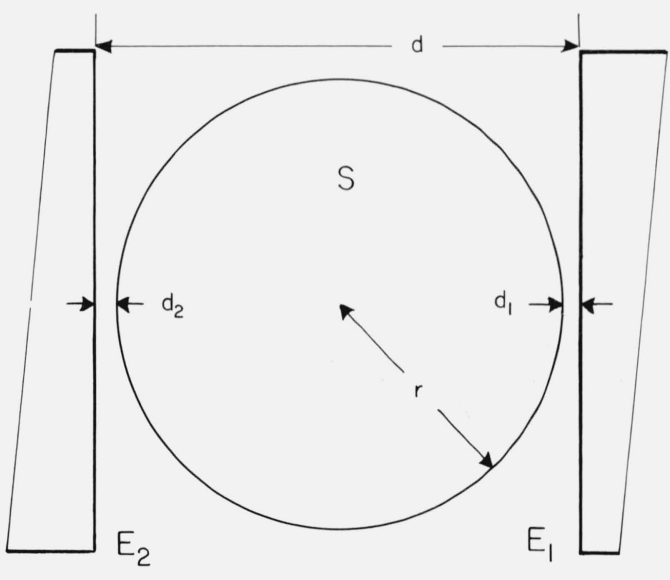

FIGURE 1. Relative position of etalon and sphere.

\section{Optics of the Interferometer}

Figure 2 shows the optical arrangement of the interferometer. A beam of light from the stabilized laser is focused to a small area, $f_{1}$, by the microscope objective, $\mathrm{L}_{3}$, and subsequently collimated by the lens $\mathrm{L}_{1}$. The collimated beam is divided in the beam splitting prism, BS, which provides a transmitted beam 


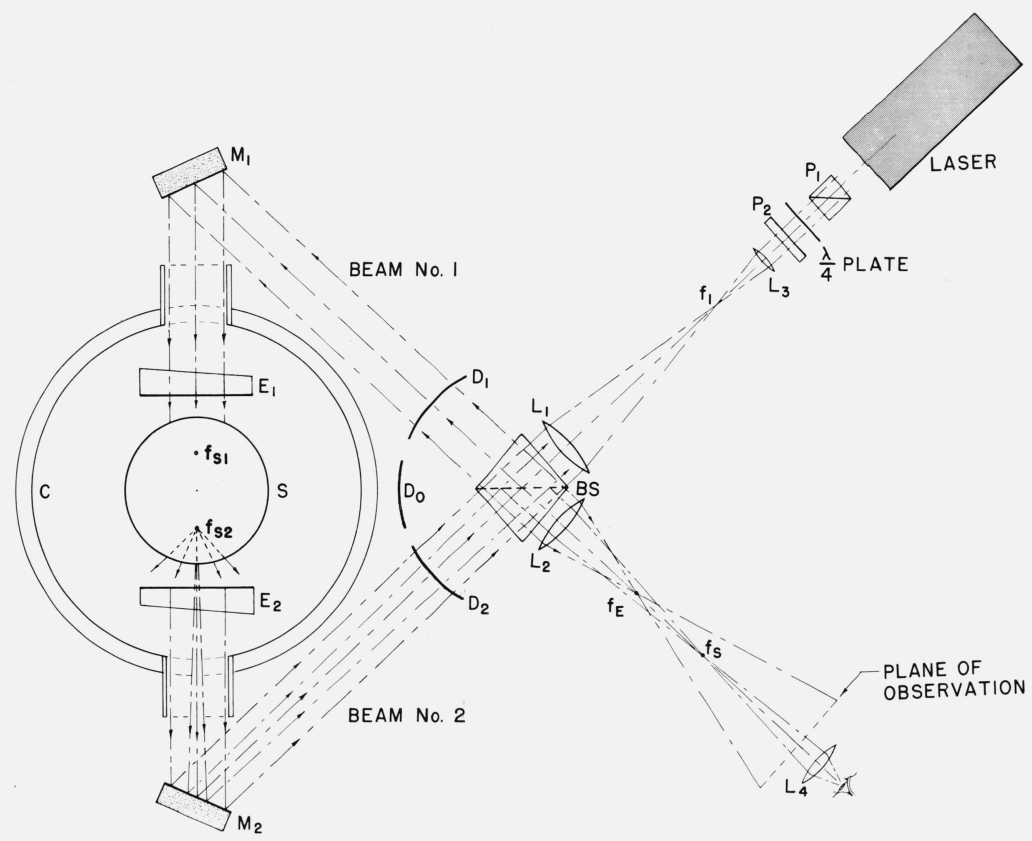

FIGURE 2. The ball and cylinder interferometer.

(No. 2) and a reflected beam (No. 1). These two beams are reflected by mirrors $M_{1}$ and $M_{2}$ toward opposite sides of the ball, $\mathrm{S}$, that is to be measured. Surfaces $E_{1}$ and $E_{2}$ form a Fabry-Perot etalon when the ball is removed. Light reflected from the two etalon surfaces returns (collimated) to $\mathrm{BS}$ and then to lens $\mathrm{L}_{2}$, which converges it to a small point, $f_{E}$, at its focus. The two beams of light then diverge from this point as concentric wave fronts, with their radii increasing and intensity (per unit area) decreasing with increasing distances beyond $f_{E}$.

The center of the ball, S, is located on the virtual, coinciding axes of lenses $\mathrm{L}_{1}$ and $\mathrm{L}_{2}$. The ball is equally distant from BS by way of the two beams. The collimated beams of light that pass through $\mathrm{E}_{1}$ and $\mathrm{E}_{2}$, which are uncoated, are then reflected from the ball and return to $\mathrm{BS}$ as divergent beams, one of which is indicated by the 3 central rays shown in beam No. 2 . These two beams (one from each side of the ball) are converged, by lens $\mathrm{L}_{2}$, to $f_{S}$ from which point they again diverge as spherical wave fronts, very similar to those from $f_{E}$.

A movable screen $\mathrm{D}_{\nu}(\nu=0,1,2)$, pivoted about the center of BS, permits the interception of beam No. 1, or No. 2 or neither beam when in positions $\mathrm{D}_{1}, \mathrm{D}_{2}$, or $\mathrm{D}_{0}$, respectively. When either beam is intercepted nonlocalized interference fringes may be observed at any point beyond $f_{s}$. The observed interferograms, shown in figure 3 , are sets of concentric, circular fringes. The order of interference at the center of the interferogram is a measure of $d_{1}$ (fig. 1), when the shutter is at $\mathrm{D}_{2}$, and $d_{2}$ when it is in position $\mathrm{D}_{1}$.

The magnification (or size) of the fringes are proportional to the distance from $f_{S}$ to the plane in which they are observed. This plane of observation is the plane of the photographic emulsion when photographing the fringes and is conjugate to the retina of the observer's eye when he is observing them. A collector lens, $L_{4}$, permits focusing of the fringes by the eye.

The contrast (visibility) of the fringes depends upon

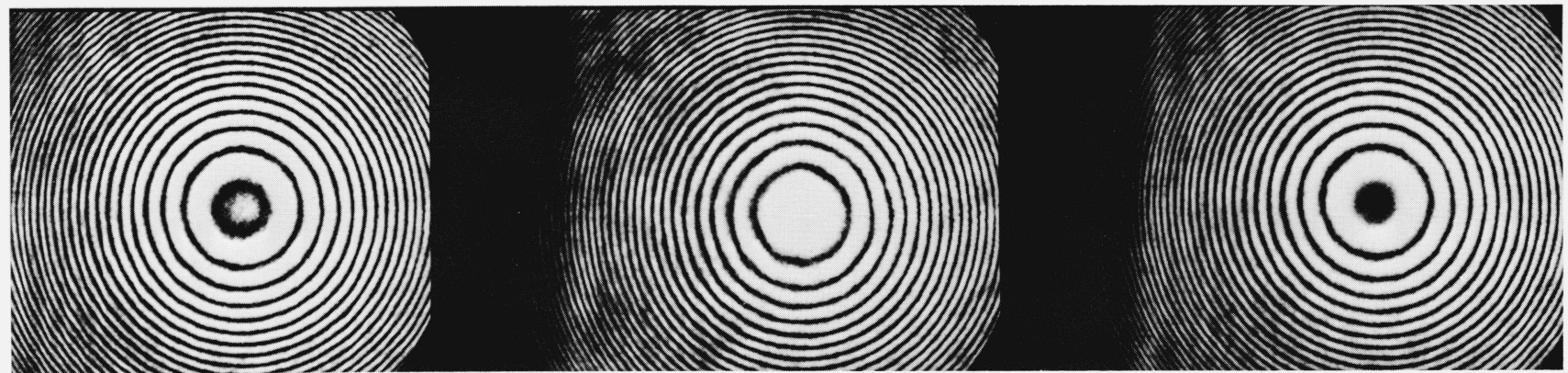

A

B

C

FIGURE 3. Interference fringes produced by light reflected from the etalon and a spherical surface. 
the relative intensity (per unit area) of the two beams. An optimum contrast and magnification is obtained by a suitable choice of reflection, transmission, spacings and powers of the optical elements. Excellent fringes were obtained for measuring steel balls (reflectivity equal 0.6) with the following dimensions and parameters:

$\mathrm{L}_{3}$ was a $4 \times$ microscope objective

$\mathrm{L}_{1}$ was a $17 \mathrm{~cm} . f .1 ., 2 \mathrm{~cm}$ diameter lens

$\mathrm{L}_{2}$ was a $25 \mathrm{~cm} . f .1 ., 2 \mathrm{~cm}$ diameter lens

BS was an imbedded aluminum film, having equal transmission and reflection

$M_{1}$ and $M_{2}$ were highly reflecting, plane mirrors

$\mathrm{E}_{1}$ and $\mathrm{E}_{2}$ were uncoated glass surfaces.

\section{Distances:}

Laser to $\mathrm{L}_{3}$ (not critical) is sufficient to allow spaces for the polarizing prism, $P_{1}$, the quarter wave plate and the parallel plate, $\mathrm{P}_{2}$, which is used to facilitate lateral adjustment of the beam;

$L_{3}$ to $L_{1}$ equals focal length of $L_{1}$ plus focal length $L_{3}$ $\mathrm{L}_{1}$ to $\mathrm{BS}=\mathrm{L}_{2}$ to $\mathrm{BS}=2 \mathrm{~cm}$

$B S$ to $M_{1}=B S$ to $M_{2}=24 \mathrm{~cm}$

$M_{1}$ to $E_{1}=M_{2}$ to $E_{2}=10 \mathrm{~cm}$

$\mathrm{L}_{2}$ to plane of observation $=250 \mathrm{~cm}$

$\mathrm{E}_{1}$ to $\mathrm{E}_{2}=6.75 \mathrm{~cm}$

diameter of $S=6.35 \mathrm{~cm}$.

\section{The Etalon}

The cavity of the etalon was chosen to provide two gaps, $d_{1}$ and $d_{2}$, between the plates and ball of approxi-

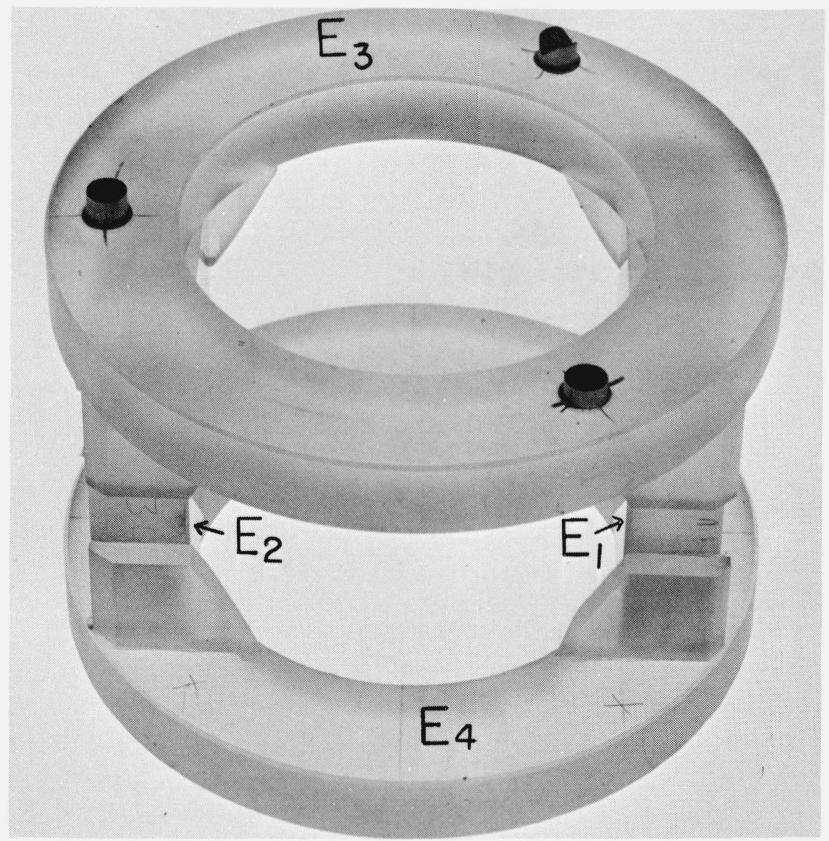

Figure 4. Photograph of the etalon.

$E_{1}$ and $E_{2}$ are surfaces. $E_{3}$ and $E_{4}$ are annular plates. mately $2 \mathrm{~mm}$ each. The etalon plates are $5 \mathrm{~cm}$ high, $5 \mathrm{~cm}$ wide, $1.3 \mathrm{~cm}$ thick, made of fused quartz and cemented to fused quartz plates $\mathrm{E}_{3}$ and $\mathrm{E}_{4}$ (see fig. 4) which serve as spacers. The annular shaped spacers have an inside diameter of $7.3 \mathrm{~cm}$ to permit insertion of the ball and an outside diameter of $11.4 \mathrm{~cm}$ for its insertion in water jacketed, brass cylinder, shown in figure 5 . The temperature of the cylinder was thermostatically controlled.

The etalon is shown upside down in figure 4. The three buttons, cemented to its base plate, are contact points that rest on three leveling screws. These screws permit vertical displacements and tilting of the etalon.

The $40^{\prime}$ wedge, between the two surfaces of each etalon place permit the elimination of light reflected from the outer surfaces. These plates were cut from a common plate to insure equality of angles and thickness. They are mounted so that when the inner surfaces are parallel the outer surfaces are also parallel to each other. Thus, when beams No. 1 and No. 2, between the etalon and mirrors $M_{1}$ and $M_{2}$, respectively, are parallel to each other the two beams, inside the etalon and before their incidence on the ball, are also parallel to each other. This insures that the rays (one of each beam), which are incident normal to the ball, will be incident at opposite ends of a common diameter. This is an essential requirement for the test and insures that

$$
d_{1}+d_{2}+2 r=d
$$

where $r$ is the radius of the ball and $d$ is the length of etalon cavity.

The alinement method, described by Bergman and Thompson $[1],{ }^{1}$ was used for paralleling the plates dur-

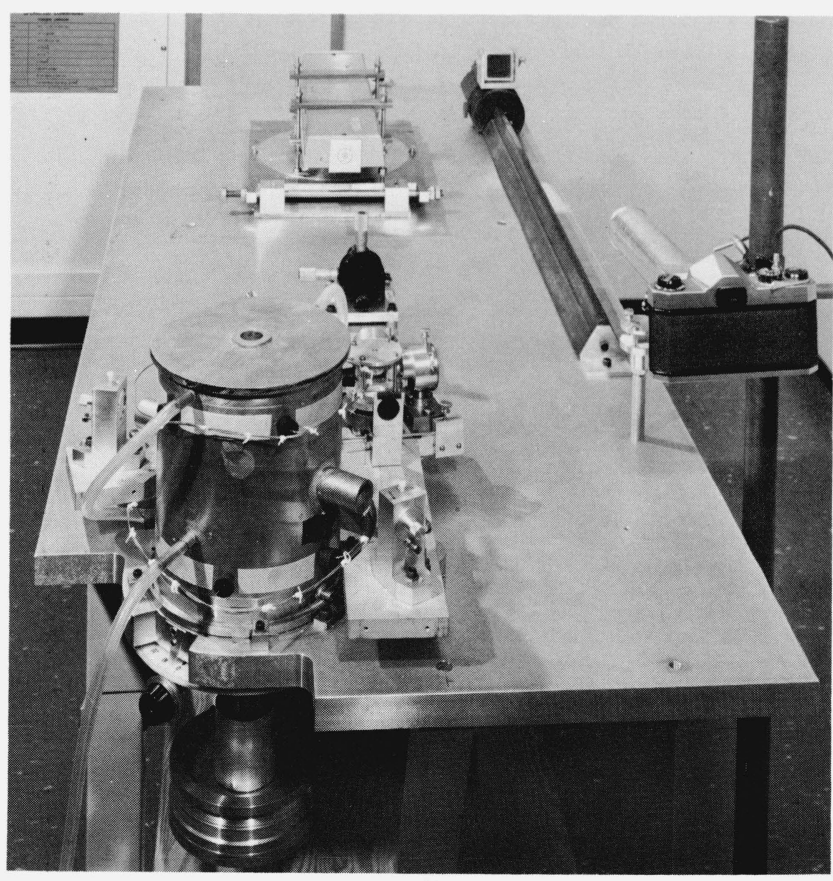

FIGURE 5. View of interferometer and accessories.

${ }^{1}$ Figures in brackets indicate the literature references at the end of this paper. 
ing the cementing of same. The resultant angle between surfaces $E_{1}$ and $E_{2}$ at the centers of the plates, was 0.3 seconds of arc. This misalinement is too small to produce any significant error in any of the measurements for which the etalon is to be used.

The conventional method for measuring etalon cavities is to determine the maximum order of interference produced by it when used (in collimated light) as a Fabry-Perot interferometer. This interferometer (see fig. 6A) does not measure the separation of the plates at any one point. The resultant cavity measurement is the average separation of the plates over the illuminated part of the aperture. This, of course, assumes the illuminating beam to be uniform in intensity. Because of imperfections [2] in polished surfaces, the difficulty (approaching the impossible) of paralleling the plates at a chosen point and the need for knowing the separation at a chosen point, the optical arrangement shown in figure 6B was used to evaluate the etalon spacing, $d$, at the point that is to be used for testing balls. The width of the fringes is controlled by adjusting the distance between the source, lens and etalon.
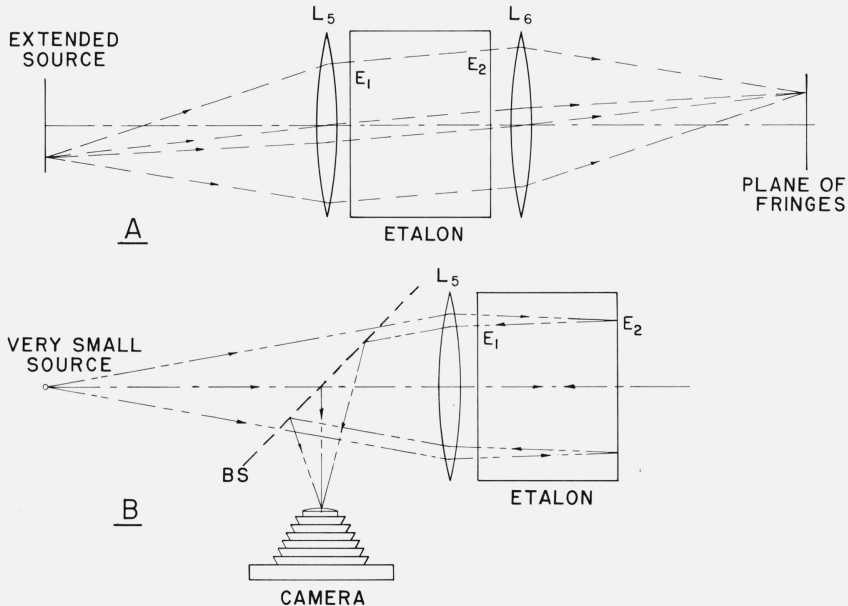

Figure 6. A. Conventional Fabry-Perot interferometer.

B. Modified Fabry-Perot interferometer for measuring the cavity at a chosen point in its aperature.

The fringes produced by the two interferometers shown in figure 6 are quite similar. Consequently, the conventional method of analyzing Fabry-Perot fringes can also be applied to those obtained from the interferometer of figure 6B. The camera, shown in figure $6 \mathrm{~B}$, is focused on a plane near the center of the etalon. The center of the fringe pattern is adjusted to the desired point, at the center of the plates. The order of interference at the conjugate of this point is then a measure of the etalon spacing at the point. Interference fringes, produced by four spectral lines of $\mathrm{Hg}$ 198, were photographed and analyzed. Because of the low illumination available with the interferometer of figure $6 \mathrm{~B}$ (with uncoated surfaces) and differences in intensity of the spectral lines used, the several lines were photographed separately. This means that the temperature of the specimen and refractive index of the air (in the etalon cavity) could be different for the several photographs. However, the accuracy of correction to standard con- ditions is no less than if all lines were photographed simultaneously. Repeated measurements yielded a value for the integral part of the etalon length (at standard conditions) that is believed to be absolutely correct. The fractional part of the order is believed to be accurate to within one tenth of an order. Even though the etalon was previously baked for stabilization, previous to the above mentioned measurements, provisions are made for remeasuring it without removing it from the temperature controlled cylinder.

If the ball, $S$, and lens, $L_{1}$, is removed from figure 2 and beam No. 1 is obstructed by the shutter in position $D_{1}$, we have the arrangements shown in figure 7 , with the dimensions altered to conserve space. As in figure 2 , we have two wave fronts diverging from points $f_{\mathrm{E} 1}$ and $f_{\mathrm{E} 2}$ which represent light reflected from $\mathrm{E}_{1}$ and $\mathrm{E}_{2}$, respectively. These wave fronts produce a set of concentric, circular, nonlocalized fringes centered on the extension of a straight line through the two points of divergence. The optical path difference between the two beams, on this line, is a measure of the length of the etalon cavity. Thus, by removing $\mathrm{L}_{1}$ the interferometer of figure 1 permits retesting the etalon with the same source used for measuring the ball-assuming the cavity changes are less than one quarter wavelength between successive measurements. Since the etalon was baked for approximately 3 days (after its assembly) at a temperature of $150{ }^{\circ} \mathrm{C}$, we do not expect much change in its dimensions.

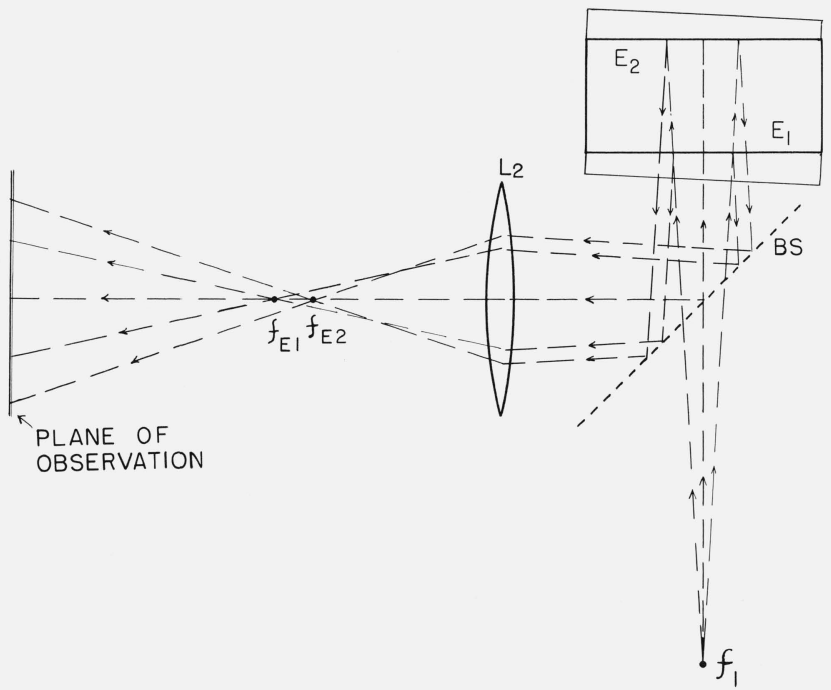

FigURE 7. Modification of ball and cylinder interferometer for measuring the etalon in situ.

\section{The Light Source}

The gas laser used with this interferometer produces a slightly divergent beam of stabilized light. This divergence was further reduced with a lens, recessed into the laser housing. Thus, a small, approximately collimated, beam of light is obtained. This improves the effects of the elements between the laser and $L_{1}$ and permits $L_{1}$ to receive the entire beam.

It is not necessary to use a stabilized laser except for retesting the etalon cavity in situ. Any gas laser is suit- 
able for measuring balls and cylinders if the etalon cavity is known and the sum $\left(d_{1}+d_{2}\right)$, does not exceed 1 $\mathrm{cm}$. To permit stabilization, however, little or no light can be allowed to return into the laser. This is readily accomplished by slightly tilting the quarter wave plate (fig. 2), the faces of prism, $\mathrm{P}_{1}$, the parallel plate, $\mathrm{P}_{2}$, and adjusting the azimuth of the polarizing prism relative to that of the quarter wave plate to eliminate all light transmitted through and returned (by reflections) to them. The polarizing prism, $\mathrm{P}_{1}$, insures that the light transmitted by it is plane polarized. The quarter wave plate changes it to circularly polarized light. The light that returns, from the interferometer, to the quarter wave plate remains approximately circularly polarized having been affected slightly by $\mathrm{BS}, \mathrm{M}_{1}$ and $\mathrm{M}_{2}$. The quarter wave plate converts the return light to approximately plane polarized light, with its plane of polarization normal to that previously transmitted by $P_{1}$, so that it is not retransmitted by $\mathrm{P}_{1}$ and little or no light is returned into the laser.

Further reduction of light, returning to the laser, is obtained by adjusting a screen with a $25 \mu \mathrm{m}$ hole (a spacial filter) so that the center of the hole coincides with $f_{1}$.

The high intensity of the laser source is important, both for obtaining short exposures of the interferograms and for adjusting the instrument. All critical adjustments are performed by observing interference fringes that are produced in various ways and with various combinations of elements. It appears practically impossible to design the hardware of this instrument so that it can be adjusted permanently to the precision which the adjustments allow and for which its operation require. Details for making adjustments are given in Appendix A, at the end of this paper.

\section{Direction of Increasing Orders}

The analysis of interferograms requires a knowledge of the direction of increasing orders of interference. The analysis of cylinder interferograms is identical to that for sphere interferograms. Consequently, this discussion will be limited to interferograms produced by spherical wave fronts.

The two sets of coherent wave fronts, $W_{1}$ and $W_{2}$ (fig. 8), combine to produce interference and the resultant interferogram is a set of concentric, circular fringes. The center of these circles ("bull's eye") is on the straight line (defined as the "axis") that passes through the centers of curvature of the two sets of wave fronts.

Let $\mathrm{P}$ (fig. 8) be any point in the plane of observation, at a distance $y$ from the bull's eye; $W_{1}$ and $W_{2}$, the wave fronts that pass through $P$, with $W_{2}$ selected as the one that has traveled the greater distance from the common source to $\mathrm{P} ; R_{1}$ and $R_{2}$, the radii of curvature, points $f_{1}$ and $f_{2}$, the centers of curvature, and $\Phi_{1}$ and $\Phi_{2}$, the phases of $\mathrm{W}_{1}$ and $\mathrm{W}_{2}$ at $f_{1}$ and $f_{2}$, respectively. Points $f_{1}$ and $f_{2}$ correspond, respectively, to $f_{\mathrm{E}}$ and $f_{\mathrm{S}}$ of figure 2 .

$R_{1}$ and $R_{2}$ are positive if the respective wave fronts

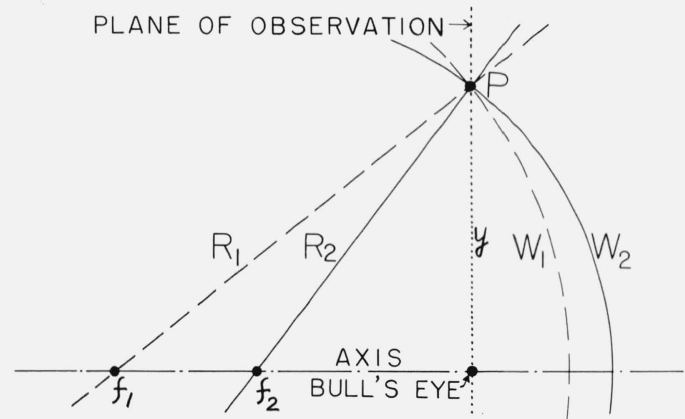

FiguRE 8. Diagram for determination of direction of increasing orders of interference.

are concave toward the incident light (diverging) and negative if convex toward it (converging).

The optical paths from the source to $\mathrm{P}$ is $\left[\Phi_{1} \gamma /\right.$ $\left.(2 \pi)+R_{1}\right]$ for $\mathbb{W}_{1}$ and $\left[\Phi_{2} \lambda /(2 \pi)+R_{2}\right]$ for $W_{2}$, where $\lambda$ is the wavelength of the light. Consequently, the order of interference $N$, at $P$ is

$$
N=\left(\Phi_{2}-\Phi_{1}\right) /(2 \pi)+\left(R_{2}-R_{1}\right) / \lambda .
$$

Since $\left(\Phi_{2}-\Phi_{1}\right)$ is constant for any interferogram, it is apparent from this equation that the order of interference increases if $\left(R_{2}-R_{1}\right)$ increases and decreases if it decreases. For all interferometers described in this paper, the plane of observation is never chosen between $f_{1}$ and $f_{2}$. Consequently, the algebraic sign of $R_{1}$ and $R_{2}$ are always the same.

It is apparent, from figure 8 , that $\left|R_{2}-R_{1}\right|$ always decreases with increasing values of $|y|$. Therefore, the algebraic sign of $\left(R_{2}-R_{1}\right)$ determines the direction of increasing orders of interference. If $\left(R_{2}-R_{1}\right)<0$ the order increases with increasing diameters $(=2 y)$ of the fringes and decreases if $\left(R_{2}-R_{1}\right)>0$.

For the analysis of interferograms produced by the interferometer shown in figure $6 \mathrm{~B}$, it is apparent that the direction of increasing order will be unchanged if the plane of observations is chosen anywhere between lens $\mathrm{L}_{5}$ and the first conjugate of the source at $f$, shown also in figure 9. It is, therefore, chosen on or to the left of $\mathrm{E}_{2}$ to avoid virtual imagery. Since the light is converging $\left(R_{1}\right.$ and $R_{2}$, negative) and $\left|R_{2}\right|$ is larger than $\left|R_{1}\right|,\left(R_{2}-R_{1}\right)>0$. Therefore, for this interferometer, the order of interference decreases with increasing diameters of the fringes.

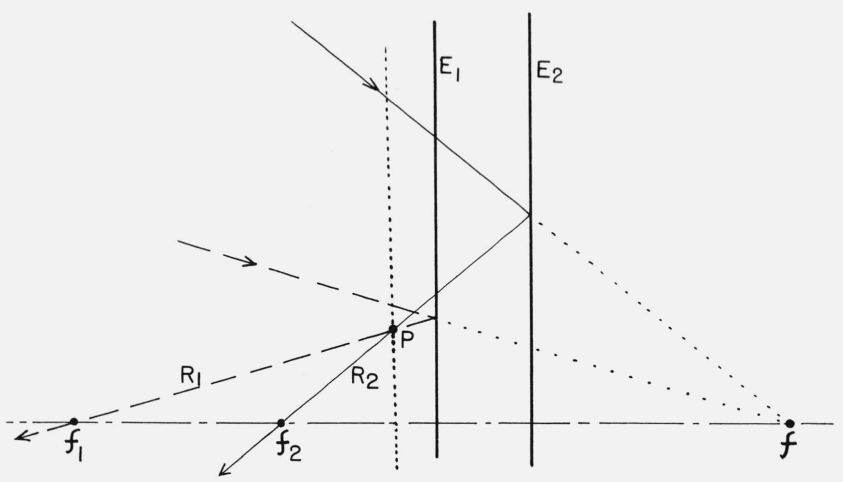

Figure 9. 
For the interferometer of figure 7 , the wave fronts are diverging at the plane of observation $\left(R_{1}\right.$ and $R_{2}$, positive) and $R_{1}<R_{2}$. Therefore, $\left(R_{2}-R_{1}\right)<0$ and the order decreases with increasing diameter of the fringes.

In the interferometer of figure 2 , the wave fronts are diverging at the plane of observation as in figure $9\left(R_{1}\right.$ and $\boldsymbol{R}_{2}$, are positive) and $R_{2}$ (the distance from $f_{s}$ to $P$ ) is smaller than $R_{1}$. Therefore $\left(R_{2}-R_{1}\right)<0$, and the order increases with increasing diameters of the fringes.

\section{Analysis of Interferograms}

If photoelectric methods of reading the fringes are used, whether at integral orders (centers of bright fringes), integral-plus-half orders (centers of dark fringes), or at other points of equal intensity, the computed value for $N$ at the bull's eye will be practically free from the procedural errors described below. If visual or photographic methods are used and readings are made either for integral or integral-plus-half orders, the observed diameters are usually in error because of variations in fringe width. This error can approximate one tenth of an order.

The solid curve in figure 10 shows the intensity distribution in a set of two-beam fringes such as the one used to produce figure 3 . The broken line curve represents the photographed density distribution and/or apparent intensity distribution when observed visually. The apparent centers of the fringes (dark or bright) are not located at maxima and minima of the intensity curve. Consequently, if the apparent centers of bright and dark fringes are considered to be at integral and integral-plus-half orders an error is introduced in the computed order at the bull's eye.

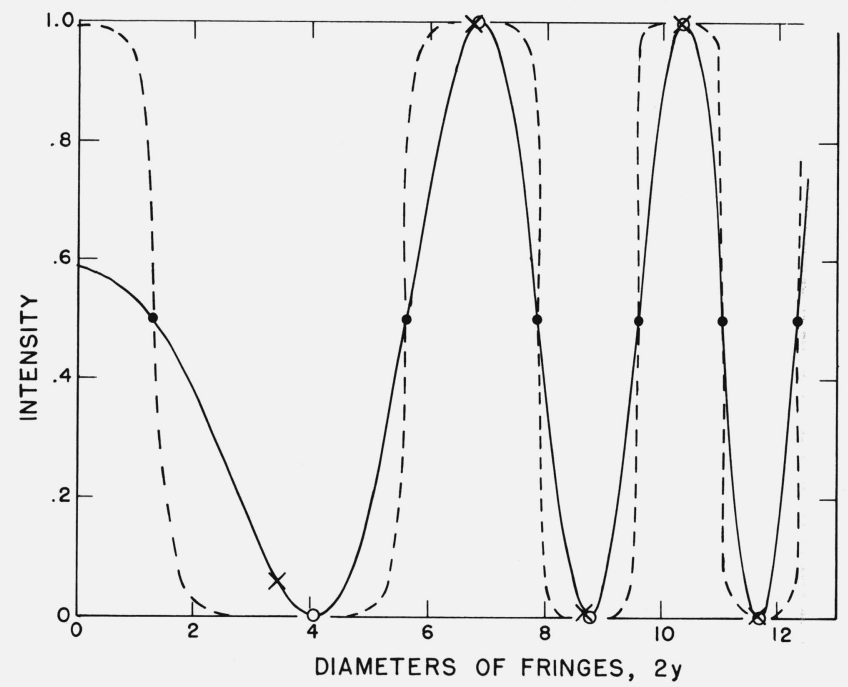

FIGURE 10. Intensity distribution of light in two-beam, Fabry. Perot type interferograms.

The small circles represent points of maximum and minimum intensity, which correspond to integral and integral-plus one-half orders of interference, respectively; the solid dots represent apparent edges of fringes, which have equal ordinates; and the X's represent apparent centers of the fringes.
The apparent edge and, consequently, also the width of a fringe, differs from one observer to another. The apparent edges of fringes in a photograph depend on the exposure and contrast of the photographic emulsion used. In general, the bright fringes will be narrower than adjacent dark fringes in an under-exposed interferogram and wider than adjacent dark fringes if the interferogram is overexposed. The relative width of the bright and dark fringes may be varied over a broad range by a choice of exposure and contrast in the photographic emulsions. However, if the aperture of the optical system is uniformly illuminated and the fringes are photographed or visually observed by any one observer, the points which he selects for edges of fringes will differ in phase from adjacent minina (or maxima) by a constant, except for accidental errors. Thus, this phase difference can be introduced into the mathematical model used for computing the orders at the bull's eye.

It can be shown that the relationship between the orders of interference, $N$, at any point, $P$, (fig. 8) and the distance, $Y$, from the bull's eye to $P$, is given, to a close approximation, by

$$
N=A+B Y^{2}+C Y^{4} .
$$

The quantity $Y$ may be replaced by $2 Y$, which is the diameter of the circle through $P$ and centered at the bull's eye. Obviously, the value of $N$ at the bull's eye, where $Y=0$, is $A$; which is the only information desired from each interferogram.

When observing for values of $Y$ in an interferogram, or a photograph of it, values of $N$ are not known at any chosen reference point. However, if the observer chooses points which he considers to be at the edges of the fringes (fig. 10), he will select points on the intensity curve where the order differs from adjacent integral orders by a constant. This constant, $E$, may be introduced into eq (2) for the statistical evaluation of $A$.

For practical reasons, the interference fringes are photographed and the negatives are used for evaluating the $Y$ 's. The dark (denser areas) fringes correspond to integral orders.

The procedure, described below for evaluating $A$, applies to all interferograms produced with this interferometer. It also applies to interferograms produced with conventional Fabry-Perot interferometers.

Observations will be made along a straight line through the bull's eye and at all edges of the first four dark fringes that have inner edges. Reference points are chosen at both outer and inner edges. When the inner edge of the innermost fringe can be read (figs. $3 \mathrm{~A}$ and $3 \mathrm{~B}$ ), the number of reference points is 16 , as shown in figure 11A; when the inner edge of the innermost dark fringe can not be read (fig. 3C), the number of reference points is 18, as shown in figure 11B. The orders of interference, $N_{\nu}$, at the chosen reference points (edges of fringes, fig. 10) are,

$$
N_{\nu}=\nu / 2+\left[1-(-1)^{\nu}\right] / 4+(-1)^{\nu} E .
$$

Where $\nu$ is the order of reading points (beginning with 

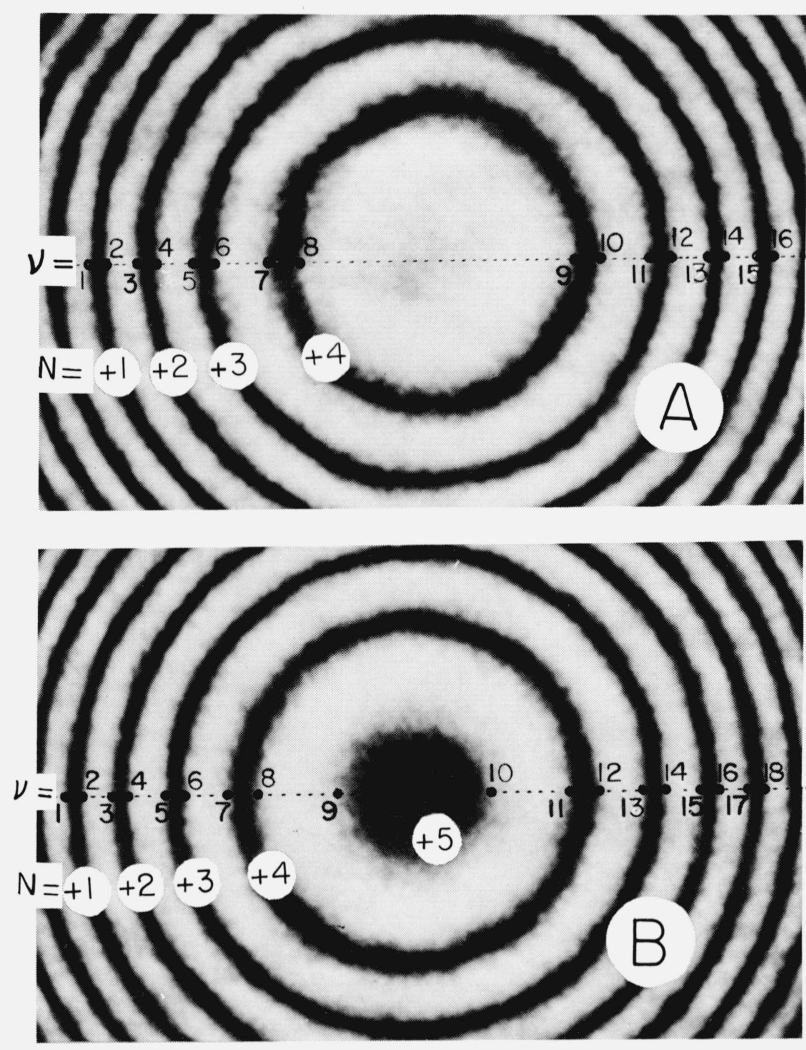

Figure 11. Enlarged portions of figures $3 B$ and $3 C$.

The numbers, in the smaller print, represent the order of reading and position of the reference points. The numbers, in the larger print, represent orders of interference.

unity at the left-hand end of the line of points) and $E$ is the unknown difference between $N_{\nu}$ and the integer closest to it.

On eliminating $N\left(=N_{\nu}\right)$ from eqs (2) and (3) we obtain,

$$
\nu / 2+\left[1-(-1)^{\nu}\right] / 4+(-1)^{\nu} E=A+B Y^{2}+C Y^{4},
$$

where $\nu=1,2,3 \ldots 8$ or 9 , respectively, for interferograms represented by figure $11 \mathrm{~A}$ or $11 \mathrm{~B}$. By writing the above set of observational equations in the form,

$$
A+B Y_{\nu}^{2}+C Y_{\nu}^{4}-(-1)^{\nu} E=\left[1+2 \nu-(-1)^{\nu}\right] / 4
$$

one can use the computational procedures of standard least squares to arrive at satisfactory values for $A, B$, $C$, and $E$.

The above procedure applies to interferograms in which the order of interference decreases with increasing values of $Y$. For interferograms in which $N$ increases with increasing values of $Y$, the plus signs before the integral values of $N$, shown in figure 11, should be replaced by negative signs. Consequently, the value of $A$ $\left(\equiv A_{i}+A_{f}=\right.$ integral plus fractional parts of $A$ ) will always be negative and the desired fractional part of the order will be the complement $\left(1-A_{f}\right)$, of $A$.

It should be noted that the spaces $d_{1}$ and $d_{2}$ can not be measured individually. Neither of these quantities are known to a sufficient approximation for applying the method of exact fractions [3]. However, the sum $\left(d_{1}+d_{2}\right)$ can be obtained sufficiently precise by measuring the quantities $d$ and $(2 r)$ in eq (1) with micrometers. The theory of exact fractions can then be applied by using the fractional parts of the orders obtained from pairs of interferograms. One interferogram, of each pair, is photographed with the shutter in position $D_{1}$ and the other with the shutter in position $D_{2}$. The sum of the fractional part of the order that represents $d_{1}$ plus the fractional part for $d_{2}$ is the sum of the fraction part of the order that represents $\left(d_{1}+d_{2}\right)$. When the corrections for phase shifts are applied, the absolute value of $2 r$ can be computed fron eq (1).

\section{Summary}

The mean diameter, and consequently also the volume, of nearly spherical balls is obtained by measuring the difference between an etalon cavity and the diameters of the ball. The etalon cavity is measured by the conventional method described in the literature - with slight modifications to obtain the separation of the plates at a known point instead of the average separation which is usually measured. The ball is placed in the cavity and the two spaces (gaps) between the ball and the etalon plates, which form two Fizeau interferometers, are obtained by the conventional methods of absolute measurement of length by interferometry. The difference between the cavity space and the sum of the two Fizeau interferometer spaces is the length of that diameter of the ball which is normal to the etalon plates. The average of many uniformly distributed diameters is a mean diameter that represents the measured volume of the ball.

The accuracy of results of measurement with this instrument is comparable to that of other precision interferometers. Its almost complete freedom from vibration, good temperature control and measurement practically confines the errors to that of determining the orders of interference. There remains in this, as in many other interferometric measurements, the question of phase shift of light when reflected from optical surfaces. If fused quartz or some other dielectric ball is used, which is recommended by this author, the resultant phase shift is the difference between internal and external reflections from dielectrics in air. If this is known the dominant error is in reading the interferograms. The spread of several values, found experimentally for a single ball diameter, did not exceed 0.05 of an order.

\section{Appendix A. A Procedure for Adjusting the Ball and Cylinder Interferometer}

This instrument is a combination of several interferometers. These are: the triangular interferometer, BS - $\mathbf{M}_{1}-\mathbf{M}_{2}$ (fig. 2), by Sagnac [4], a Fabry-Perot interferometer [5], $\mathrm{E}_{1}-\mathrm{E}_{2}$; and two Fizeau interferometers [6], $E_{1}-S$ and $E_{2}-S$. It is essential that the two beams of light, No. 1 and No. 2, be parallel to each other in the etalon cavity. Only then can $E_{1}$ and $E_{2}$ be simultaneously adjusted normal to the two 
beams of light. Also, it is important to have each pair of component rays (one in each beam, No. 1 and No. 2) of the Sagnac interferometer return to a common point in BS and pass through a common point $f_{\mathrm{E}}$. Fortunately, this interferometer permits adjustments that insure this performance.

When two or more interferometers are compounded, the adjustments of either one effect the resultant output. Consequently, precision adjustment of the combination requires successive approximations. This requires that the adjuster become acquainted with all of the adjustments. For instance, when adjusting beams No. 1 and No. 2 into coincidence, a rotation of BS has relatively little effect on beam No. 2 and the displacement of beam No. 1 is proportional to the distance from BS. A rotation of $M_{1}$ changes the relative position of the two beams more at $D_{2}$ than at $D_{1}$. The elements BS, $L_{1}, L_{2}, L_{3}$, and $M_{1}$, and $M_{2}$ are mounted on and fastened to a flat, rigid base. A line is engraved on this base to indicate the normal projection of the center of the light beams. The centers of the elements are mounted two inches vertically above the horizontal base plate and fine adjustments are performed by relatively large screws that permit flexing the bases of the mountings of each element.

A lucite triangle, shown in figure 12 , is used for locating the position of the two light beams over any point at which the indexing point of the triangle is located. Two lines are engraved on the triangle. One line is approximately 3 in long, parallel to one leg of the triangle and extending to the other leg. This line, when indexed with the engraved line on the base plate, serves to place the line vertically above the base plate line. A second short line is engraved on the triangle, 2 in from the indexing end of the lst mentioned line and normal to it. When the triangle is indexed with the base plate line and standing vertically, the intersection of its two lines indicates the desired position of the centers of the light beams. The surfaces of lucite, when slight abraded, will scatter enough light to show the positions of the two beams from either side.

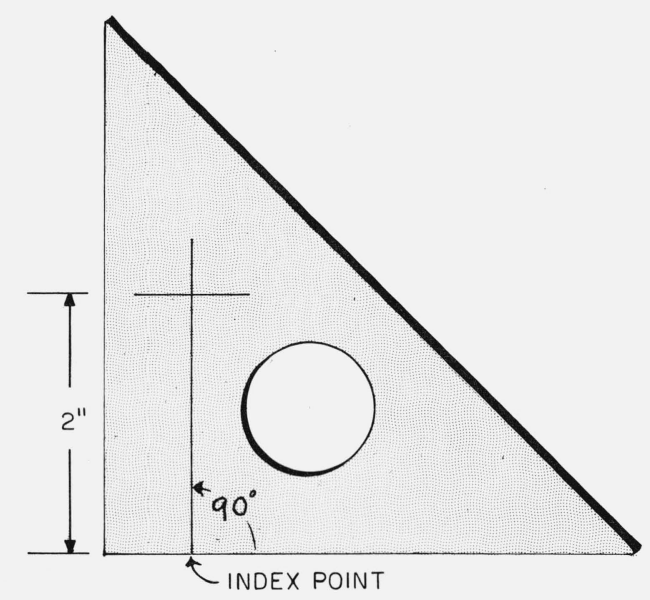

FIGURE 12. Interferometer beam locator.
To aid in making adjustments, it will be convenient to cement to the laser housing (fig. 5) an opaque screen black on the inner side and white on the outer side, with a circular aperture (or hole) at its center that is slightly larger than the light beam. This screen should be normal to the beam and adjusted laterally to center the light beam in the aperture.

The following is a recommended procedure for adjusting the ball and cylinder interferometer:

Step 1 . The laser is placed approximately $70 \mathrm{~cm}$ from BS, to allow adequate space for other elements shown in figure 2, with the center of the laser beam coinciding with the centers of $\mathrm{BS}$ and $\mathrm{M}_{2}$.

Step 2. Remove $\mathrm{L}_{1}, \mathrm{~L}_{3}$, and $\mathrm{S}$ from the interferometer and place the etalon as shown in figure 2. The surfaces, $\mathrm{E}_{1}$ and $\mathrm{E}_{2}$ should be approximately normal to the line joining the centers of $M_{1}$ and $M_{2}$, with this line passing near the centers of $E_{1}$ and $E_{2}$. Adjust the shutter to position $\mathrm{D}_{0}$. Adjust $\mathrm{BS}$ to direct beam No. 1 to the center of $M_{1}$. Adjust $M_{1}$ to direct beam No. 1 to the center of $\mathrm{M}_{2}$. Adjust $\mathrm{M}_{2}$ to direct beam No. 2 to the center of $\mathrm{M}_{1}$. The two beams will each return to BS where each is again divided, so that two components return toward the laser and two components are transmitted toward the observer. Each pair of these component beams will emerge from BS accurately parallel to each other but, in general, not coincident.

Step 3. The four emerging component beams are adjusted to coincide in pairs by the following procedure:

Place an extended, brightly illuminated screen between BS and the laser, with a small black spot (or an $\mathbf{x}$ ) on it near the optic axis of the interferometer. On looking at the black spot through BS, from the eye position (shown in fig. 2), with $\mathrm{L}_{2}$ and $\mathrm{L}_{4}$ removed, one sees two images of it. The observed separation of these images indicate the magnitude and direction of separation of each pair of component rays from each and every point on the source. These images are formed by light (beams No. 1 and No. 2) traversing the triangle, BS, $\mathrm{M}_{1}, \mathrm{M}_{2}$, in opposite directions. The two images can be adjusted into coincidence by either $\mathrm{BS}, \mathrm{M}_{1}$ or $\mathbf{M}_{2}$. These adjustments also affect the position of the beams relative to each other and to the elements of the interferometer. Usually an adjustment (turning of one screw) can be found that improves both the position of the two beams (as revealed by the triangle of fig. 12) and the two source images. When the two images are adjusted to near coincidence, white light fringes of interference can be seen in the source. The width of these fringes indicates the magnitude and the direction of the fringes indicates the direction of separation of the two images of the source. Further adjustment of $M_{1}$ or $M_{2}$, while observing the white light fringes, will produce one broad, fluffed out fringe which indicates exact coincidence of the images. When the two images of the source are adjusted to coincide, all pairs of components (one from each of beams No. 1 and No. 2) will emerge from BS in coincidence.

At this stage of the adjustment procedure, the etalon surfaces $E_{1}$ and $E_{2}$ can be adjusted normal to any one component ray but in general not to any pair of com- 
ponents because component pairs, although parallel and coincident after emerging from the interferometer, are neither parallel or coincident in the etalon cavity.

Step 4. The telescopic combination, $\mathrm{L}_{1}, \mathrm{~L}_{3}$, is now placed in position, with $\mathrm{L}_{1}$ close to $\mathrm{BS}$ (for conservation of space) and adjusted to pass collimated light into BS. If the axes of $L_{1}$ and $L_{3}$ are coincident, the unit can be adjusted to leave the paraxial ray unchanged in direction and position. The expansion of the beam, by this unit, must be large enough to provide adequate uniformity of illumination in the fringe patterns, over the area that will be used for measurements. Excessive expansion of the beam results in unnecessary loss of light.

The adjustment of $E_{1}$ and $E_{2}$ normal to the two component beams is affected in two steps (steps 5 and 6). First, the etalon is adjusted to reflect the two component beams parallel (but in opposite direction) to each other and, secondly, they are adjusted (simultaneously) normal to $E_{1}$ and $E_{2}$ by changing the direction of the incident beams.

Step 5 . The two beams are obstructed by placing an opaque screen between $\mathrm{E}_{1}$ and $\mathrm{E}_{2}$. Also, a white screen should be located between the indicated positions of $\mathrm{L}_{2}$ and $\mathrm{L}_{4}$, for receiving the four component beams reflected from the four surfaces of the two etalon plates. There will also be four similar beams that return toward the laser. The screen, described above, through whose aperture the light leaves the laser, serves to indicate the position of these returning beams relative to the source. On rotating the etalon the four beams (on each screen) move together in pairs. Each pair represents light reflected from the two surfaces of one etalon plate. The two pairs move in opposite directions on both screens. Each beam must be identified with the surface from which it is reflected. The shutter D permits identification of the plate and wetting the etalon surface $\left(\mathrm{E}_{1}\right.$ or $\left.\mathrm{E}_{2}\right)$ permits identification of the individual surfaces.

Step 6. The two beams from $E_{1}$ and $E_{2}$ are adjusted to coincide with each other by rotation of the etalon. Rotation of the etalon about a horizontal axis, normal to the beam, causes the four images to move vertically on the screen near $f_{\mathrm{E}}$. When the two beams from the two inner surfaces are adjusted to near coincidence, interference fringes will appear on the screen in the area illuminated by them. The direction and width of these fringes indicates what direction of rotation of the etalon is required to broaden the fringes. When the fringes are broadened to portray a single fringe on the screen, the etalon is adjusted to the desired condition. It can be shown, when the above adjustment is completed, that the two component beams are incident at equal angles on $E_{1}$ and $E_{2}$. This angle of incident can now be reduced to zero by changing the direction of the collimated beam from the laser. This is effected by moving the laser beam toward the two coinciding images, that are reflected from $E_{1}$ and $E_{2}$ and return to the screen cemented to the laser housing. If this movement is effected while maintaining the beam directed toward the center of $\mathrm{L}_{1}$, the beams from $\mathrm{E}_{1}$ and $E_{2}$ will approach the aperture in the screen. When the two beams return to the source, they are each normal to $E_{1}$ and $E_{2}$. Because of the magnification of the telescopic system, $\mathrm{L}_{1}, \mathrm{~L}_{3}$, and the relatively large distance from $L_{3}$ to the screen on the laser, the movement of images on this screen is very sensitive to rotational adjustments of the etalon. The beams will be sufficiently near normal to $E_{1}$ and $E_{2}$ when the beams return to within one millimeter of the center of the aperture in the laser screen.

Step 7. If the obstructing screen, between $E_{1}$ and $\mathrm{E}_{2}$ is removed, the two beams transmitted by the etalon, may now be used to recheck the degree of coincidence of component rays that emerge from the interferometer. If the above described adjustments, for making all rays normal to $E_{1}$ and $E_{2}$, were appreciable, the resultant rotation of the etalon will have displaced the two transmitted beams laterally and in opposite directions so that they no longer coincide after returning to BS. A repetition of step 3 will restore the coincidence of all emerging component rays. This may be done without removing $L_{1}$ and $L_{3}$ from the system. The extended screen should be located close to $L_{1}$ for best visibility of the white light fringes (assuming the screen to be illuminated with white light)

Step 8. Repeat step 5. Usually, the magnitude of this adjustment will be much less than was required previously.

Step 9. Repeat step 3 if the white light fringes have been narrowed too much.

A few alternating repetitions of steps 3 and 5 should bring these adjustments to satisfactory approximation.

With collimated light from the laser traversing the etalon, the ball can then be adjusted to its proper position. This consists of locating the center of the ball on the axis (center of the collimated beam) and at a point equally distant from BS by way of $\mathrm{M}_{1}$ and $\mathrm{M}_{2}$.

Step 10. Place the ball at its approximate proper position. If $\mathrm{E}_{1}$ and $\mathrm{E}_{2}$ have been located equally distant from BS by way of $M_{1}$ and $M_{2}$, respectively, the ball should be centered in the etalon. A test for the lateral position of the ball is to place a sheet of white paper, having a circular hole in it whose diameter equals that of the light beam, between the etalon and $M_{1}$ (or $M_{2}$ ) so that the beam of collimated light coincides with the hole. If the ball is not centered in the beam, the light reflected from the ball will not illuminate the paper screen symmetrically about the hole. When this illumination is symmetrical about the hole, the ball is on axis. The two beams of light, returning to BS from opposite sides of the ball, will also illuminate the screen near $f_{\mathrm{S}}$, producing a set of concentric, circular fringes of interference. The width or frequency of these fringes is a measure of the difference between the two distances, $\mathrm{S}, \mathrm{M}_{1}, \mathrm{BS}$ and $\mathrm{S}, \mathrm{M}_{2}, \mathrm{BS}$. Moving the ball parallel to the axis (line through the centers of $M_{1}$ and $\mathrm{M}_{2}$ ) changes the width of the fringes. When the fringes become very broad, the two distances are very near equal.

Step 11. Lens $\mathrm{L}_{2}$ is mounted, with its axis coinciding with the center of the beams of light from BS. With $\mathrm{D}$ in the position $\mathrm{D}_{1}$ (fig. 2) the collimated light from $\mathrm{E}_{1}$ 
will be focused at $f_{E}$ and the divergent light from the ball will be focused at $f_{\mathrm{s}}$. The two beams diverge from these two points, producing interference on a screen located at any place beyond $f_{\mathrm{s}}$. These fringes may be observed in any plane with a collector lens $\mathrm{L}_{4}$ or photographically recorded by allowing the two beams to fall on a sheet of photographic emulsion, placed normal to the line through $f_{E}$ and $f_{S}$, at any point beyond $f_{\mathrm{S}}$. The order of interference at the center of this interferogram is a measure of the distance $d_{1}$, shown in figure 4. On shifting the shutter to position $\mathrm{D}_{2}$, a similar interferogram is obtained which represents the distance $d_{2}$.

Since the beams of light from $\mathrm{S}, \mathrm{E}_{1}$ and $\mathrm{E}_{2}$ are comparable in brightness with those beams reflected from the outer surfaces of the etalon and the outer surfaces of BS, the two beams that produce the desired interference must be isolated from the others. This is effected by constructing the two etalon plates and the two prisms that form BS, with angles between their respective surfaces sufficiently large to cause the undesirable beams to diverge sufficient for easy elimination. An opaque screen, located between $f_{\mathrm{S}}$ and $f_{\mathrm{E}}$, with an aperture just large enough to transmit the two desired beams, will eliminate all the other beams.

In practice, a small reflecting prism, located between $f_{\mathrm{S}}$ and $f_{\mathrm{E}}$, reflects the two beams (eliminating others by its small size) parallel to an optical bench (shown in fig. 5), onto a mirror that returns them to a camera box. The optical bench serves to reduce space by folding the light in such a manner that the path can be varied from 0.5 to 2.5 meters and yet be limited to the top of a 2 x 6-foot table.

\section{References}

[1] Bergman, T. G., and J. L. Thompson, Applied Optics, 7, p. 923 (1968).

[2] Saunders, James B., J. Res. Nat. Bur. Stand (U.S.), 47, No. 3, 148-155 (Sept. 1951) RP2239.

[3] Perot, A., and Ch. Fabry, Ann. Chem. Phys. 16, p. 289 (1899).

[4] Sagnax, M. G., Acasmie des Sciences, Seance du 23 Mai, 1910, p. 1302.

[5] Fabry, C., and A. Perot, Ann. Chem. Phys. (7) 16 (1899), p. 115.

[6] Fizeau, H., Ann. Chem. Phys. (3) 66 (1862), p. 429.

(Paper 76C1\&2-327) 